\title{
Fruquintinib: a novel antivascular endothelial growth factor receptor tyrosine kinase inhibitor for the treatment of metastatic colorectal cancer
}

This article was published in the following Dove Press journal:

Cancer Management and Research

\author{
Ying Zhang' \\ Jia-Yun Zou' \\ Zhe Wang ${ }^{2}$ \\ Ying Wang' \\ 'Department of Oncology, Shengjing \\ Hospital of China Medical University, \\ Shenyang, Liaoning Province, People's \\ Republic of China; ${ }^{2}$ Department of \\ Pathology, Shengjing Hospital of China \\ Medical University, Shenyang, Liaoning \\ Province, People's Republic of China
}

Correspondence: Ying Wang Department of Oncology, Shengjing Hospital of China Medical University, 39 Huaxiang Street, Tiexi District, Shenyang, Liaoning Province, People's Republic of China

Tel/fax +8624966I56 33II

Email wang_ying@sj-hospital.org

\begin{abstract}
Angiogenesis plays a critical role in the neoplastic growth, progression, and metastasis of colorectal cancer (CRC) in a process regulated by vascular endothelial growth factor (VEGF) family members and their receptors (VEGFR). Several small-molecule antiVEGFR tyrosine kinase inhibitors (TKIs), such as regorafenib, famitinib, axitinib and apatinib, have been shown to be effective in treating metastatic colorectal cancer (mCRC). Fruquintinib (ELUNATE ${ }^{\circledR}$ ) is a novel oral anti-VEGFR TKI, originated and developed by Hutchison MediPharma. Fruquintinib is a potent and highly selective small-molecule inhibitor of VEGFR-1, -2 and -3 . In the Phase 3 FRESCO trial, fruquintinib improved both overall survival (OS) and progression-free survival (PFS) in patients with mCRC, compared with placebo. Fruquintinib also showed an acceptable safety and tolerability profile. Based on the data from this trial, fruquintinib was approved by the China Food and Drug Administration (CFDA) in 2018, for the treatment of patients with mCRC who had undergone at least two prior standard anticancer therapies. The existing clinical trials and future prospects of fruquintinib in $\mathrm{mCRC}$ will be discussed in this article. In addition, to better understand the role of fruquintinib in this setting, recent advances in other anti-VEGFR TKIs for mCRC treatment are also reviewed herein.
\end{abstract}

Keywords: fruquintinib, VEGFR, tyrosine kinase inhibitor, metastatic colorectal cancer

\section{Introduction}

$\mathrm{CRC}$ is the third most common cancer and the fourth leading cause of cancer death worldwide, accounting for nearly 1.4 million new cases and almost 700,000 deaths in 2012. ${ }^{1}$ Based on demographic projections and temporal profiles, the global number of CRC cases is expected to increase to more than 2.2 million new cases and 1.1 million cancer deaths per annum by $2030 .{ }^{1}$ Increases in the morbidity and mortality of $\mathrm{CRC}$ is related to current levels of human development; changes in incidence may reflect the adoption of more Western lifestyles. ${ }^{2}$

Approximately $25 \%$ of patients have $\mathrm{mCRC}$ at the first diagnosis and nearly $50 \%$ of patients who undergo surgery for early-stage CRC eventually develop mCRC. ${ }^{3}$ Common chemotherapy drugs for mCRC consist mainly of 5-fluorouracil, oxaliplatin and irinotecan. Combination of two chemotherapy drugs is associated with 20 months OS, while the addition of VEGF and epidermal growth factor receptor (EGFR) inhibitors, could extend OS to nearly 30 months. $^{4-7}$

Unfortunately, the global outcome of $\mathrm{mCRC}$ is still poor and new therapies are urgently needed. Fruquintinib is a novel small molecule anti-VEGFR TKI that 
targets VEGFRs (VEGFR-1, -2 and -3 ), and has been used to treat mCRC. This review will summarize and discuss the trials of fruquintinib.

\section{VEGFR family TKIs}

Angiogenesis plays a critical role in tumor cell proliferation, vascular remodeling, cell dissemination and metastasis. The angiogenic process is mainly regulated by the interactions between soluble VEGFs and their receptors (VEGFRs). ${ }^{8,9}$ VEGFs consist of five glycoproteins (VEGF-A, VEGF-B, VEGF-C, VEGF-D, and placenta growth factor). VEGFRs include three types of cell membrane receptors: VEGFR-1 (Flt-1), VEGFR-2 (Flk-1/ KDR) and VEGFR-3 (Flt-4). The molecular structure of VEGFRs is comprised of an extracellular domain (ligandbinding domain), a transmembrane domain, and an intracellular region containing a tyrosine kinase domain. ${ }^{10}$ Among the three receptors, VEGFR-2 is a receptor for VEGF-A, which is prominently involved in the over-formation of blood vessels. The interaction between VEGF-A and VEGFR-2 leads to activation of intracellular signaling cascades promoting endothelial cell proliferation and migration. $^{11,12}$ VEGFR-1 is a receptor for VEGF-A, VEGF-B and placental growth factor. VEGFR-1 also regulates angiogenesis but shows an inhibitory effect on KDR-mediated proliferation. ${ }^{13,14}$ VEGFR-3 is a receptor with a high affinity for VEGF-C and VEGF-D, angiogenic factors that mediate lymphangiogenesis. VEGFR-3 is only expressed in lymphatic vessels and endothelial cells, and is involved in the dissemination of tumor cells to regional lymph nodes. ${ }^{15}$ One study examining the heterogeneous expression of VEGFRs in human CRC tissues demonstrated that all three VEGFRs play different roles in tumor vascularization. ${ }^{16}$

Small-molecule anti-VEGFR TKIs have been shown to be effective in a variety of malignancies and are characterized by favorable oral bioavailability and the ability to target VEGFRs involved in tumor angiogenesis and growth. ${ }^{17}$ Herein, some TKIs that have been studied in mCRC are listed. First-generation anti-VEGFR TKIs, such as sorafenib, sunitinib, vatalanib, cabozantinib, brivanib, cediranib, linifanib, lenvatinib, nintedanib, regorafenib and famitinib, can inhibit VEGFR and other receptors involved in angiogenic signaling with similar potency. At the maximum tolerated dose (MTD), exposure of the multi-target TKI is limited. Since all targets are inhibited, the duration of inhibition for any target, particularly VEGFR, becomes less optimal and/or short. Thus, second-generation anti-VEGFR TKIs, such as axitinib, apatinib, tivozanib and fruquintinib, are characterized by potent and highly selective inhibition of VEGFR, which is expected to maintain target inhibition while minimizing off-target toxicity and providing high drug exposure at the MTD. ${ }^{17,18}$

\section{Fruquintinib: a novel anti-VEGFR TKI}

Fruquintinib (6-[6, 7-dimethoxyquinazolin-4-yloxy]-N, 2-dimethylbenzofuran-3-carboxamide) is a novel, smallmolecule, oral anti-VEGFR TKI, with high affinity for VEGFR-1, -2 and -3 . Fruquintinib has been widely used to inhibit tumor angiogenesis in vivo and in vitro. ${ }^{19}$ Molecular targets of fruquintinib are shown in Figure 1.

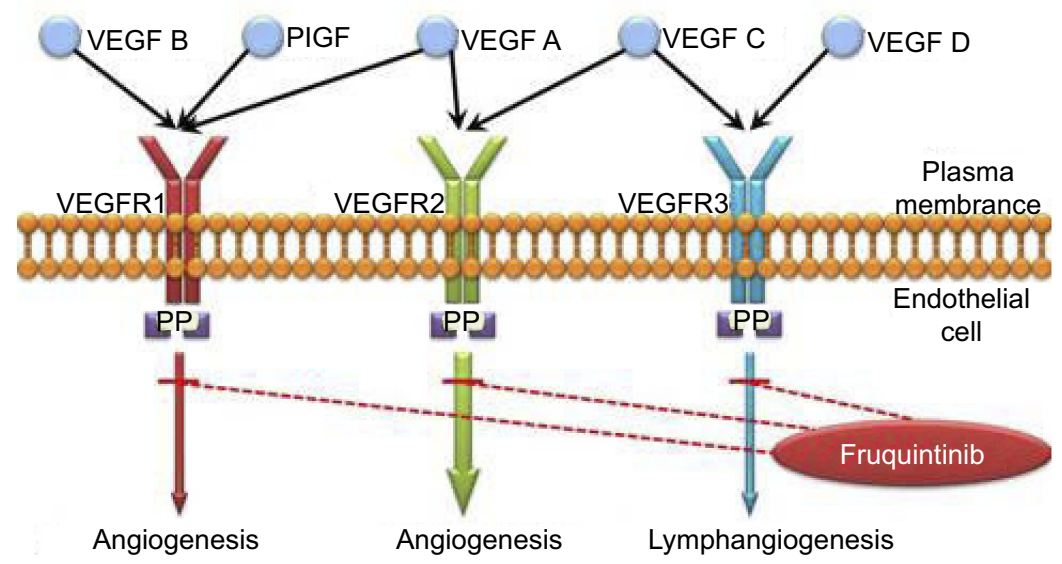

Figure I Molecular targets of fruquintinib. 
Fruquintinib (ELUNATE ${ }^{\circledR}$ ) was originated and developed by Hutchison MediPharma and then co-developed and commercialized by Eli Lilly and Company in October 2013 under a licensing agreement in China. ${ }^{20}$ On September 4, 2018, fruquintinib was granted approval by CFDA to treat patients with $\mathrm{mCRC}$ who had failed at least two prior standard antitumor therapies, including fluoropyrimidine, oxaliplatin and irinotecan, with or without prior use of anti-VEGF or anti-epidermal growth factor receptor (EGFR) treatment. ${ }^{21}$ Target differences between fruquintinib and other VEGFR TKIs are summarized in Table 1.

\section{Pharmacodynamics and pharmacokinetics}

\section{Pharmacodynamics}

Fruquintinib exhibits excellent antitumor activity, both in vitro and in vivo. ${ }^{20}$

In vitro, fruquintinib has been shown to inhibit the activity of a panel of 253 kinases. Fruquintinib showed potent inhibition of VEGFR-1, -2 and -3 (half maximal inhibitory concentration $\left[\mathrm{IC}_{50}\right]$ values were $33 \mathrm{nmol} / \mathrm{L}, 35 \mathrm{nmol} / \mathrm{L}$ and $0.5 \mathrm{nmol} / \mathrm{L}$, respectively), and weak inhibition of RET, FGFR-1 and c-KIT kinases. In addition, fruquintinib also inhibits endothelial cell proliferation and tubule sprouting, thereby preventing tumor angiogenesis. ${ }^{20}$

In vivo, the anti-tumor activity of fruquintinib was evaluated in several established human tumor xenograft models, such as colon cancer, gastric cancer and lung cancer. Treatment with the combination of fruquintinib and other chemotherapy drugs, or treatment with fruquintinib alone, showed good antitumor efficacy. A study in the HT-29 colon cancer model indicated that the antitumor activity of fruquintinib alone was moderately sensitive. In another study using a xenograft model with cells derived from human colon cancer patients, treatment with fruquintinib and oxaliplatin showed a potent inhibitory effect, with a tumor growth inhibition (TGI) of $68 \%$, compared with TGIs of $36 \%$ and $23 \%$ for fruquintinib and oxaliplatin alone, respectively. ${ }^{20}$

\section{Pharmacokinetics}

The pharmacokinetics (PK) of fruquintinib were estimated in preclinical and clinical studies. ${ }^{36-38}$

In a preclinical study, fruquintinib exhibited good PK characteristics, including rapid absorption and high oral bioavailability, slow elimination, reasonable tissue distribution and a low risk of drug-drug interactions. ${ }^{36}$

Two clinical trials were conducted to estimate the human PK of fruquintinib in Chinese patients. In a phase I trial, the plasma concentration of fruquintinib increased rapidly after 2 to $4 \mathrm{hrs}$ (h) of administration. As the dose of fruquintinib was increased from $1 \mathrm{mg}$ to $6 \mathrm{mg}$, the peak plasma concentration $\left(\mathrm{C}_{\max }\right)$ and area under the concentration-time curve to $24 \mathrm{~h}$ post-dose $\left(\mathrm{AUC}_{0-24 \mathrm{~h}}\right)$ increased proportionally to the dose. For instance, in a continuous regimen of $4 \mathrm{mg}$ once daily, the mean $C_{\max }$ and $\mathrm{AUC}_{0-24 \mathrm{~h}}$ of fruquintinib was $290 \pm 61.1 \mathrm{ng} / \mathrm{mL}$ and $5212 \pm 1227 \mathrm{ng} . \mathrm{h} /$ $\mathrm{mL}$, respectively. When the dose was increased to $6 \mathrm{mg}$ once daily, the mean $C_{\max }$ and $\mathrm{AUC}_{0-24 \mathrm{~h}}$ were increased to $508 \pm 63.6 \mathrm{ng} / \mathrm{mL}$ and $9230 \pm 1215 \mathrm{n} . \mathrm{gh} / \mathrm{mL}$, respectively. Fruquintinib showed a moderately long terminal half-life $\left(T_{1 / 2}\right)$ of $42 \mathrm{~h}$ and in a continuous regimen, the plasma concentration stabilized after 14 days of dosing. In a 3week-on/1-week-off regimen, it was observed that the plasma concentration of fruquintinib was stable after 14 days' administration, remained stable until the end of the 21st day of administration, and was then almost completely eliminated after 7 days of withdrawal. Based on the above PK parameters and the evaluation of efficacy, the study recommended a continuous regimen of $4 \mathrm{mg}$ once daily or a 3-week-on/1-week-off regimen of $5 \mathrm{mg}$ once daily. ${ }^{37}$

In another phase I tiral, 6 healthy male volunteers were given a single oral dose of $5 \mathrm{mg}{ }^{14} \mathrm{C}$-labeled fruquintinib. The results also showed that fruquintinib had favorable PK characteristics, which could be rapidly absorbed $\left(C_{\max }\right.$ $113 \mathrm{ng} / \mathrm{mL}$ and time to peak plasma concentration $\left[T_{\max }\right]$ $2 \mathrm{~h}$ ) and slowly eliminated ( $\left.T_{1 / 2} 33.4 \mathrm{~h}\right)$. In the liver, fruquintinib was widely metabolized by various enzymes. In plasma, the unaltered parent compound was the predominant radioactive component and M11 was the major circulating metabolite. After metabolism, only a small amount of the parent compound was excreted in the urine or feces (less than $6 \%$ of the administered dose), and urine was the major route of excretion. ${ }^{38}$

\section{Clinical trials in $\mathrm{mCRC}$ Clinical trials of fruquintinib}

Three clinical trials have been used to evaluate the efficacy and safety of fruquintinib monotherapy for mCRC. ${ }^{39,40} \mathrm{In}$ all three trials, fruquintinib was administered in a 3-weekon/1-week-off regimen of $5 \mathrm{mg}$ once daily. Best supportive 


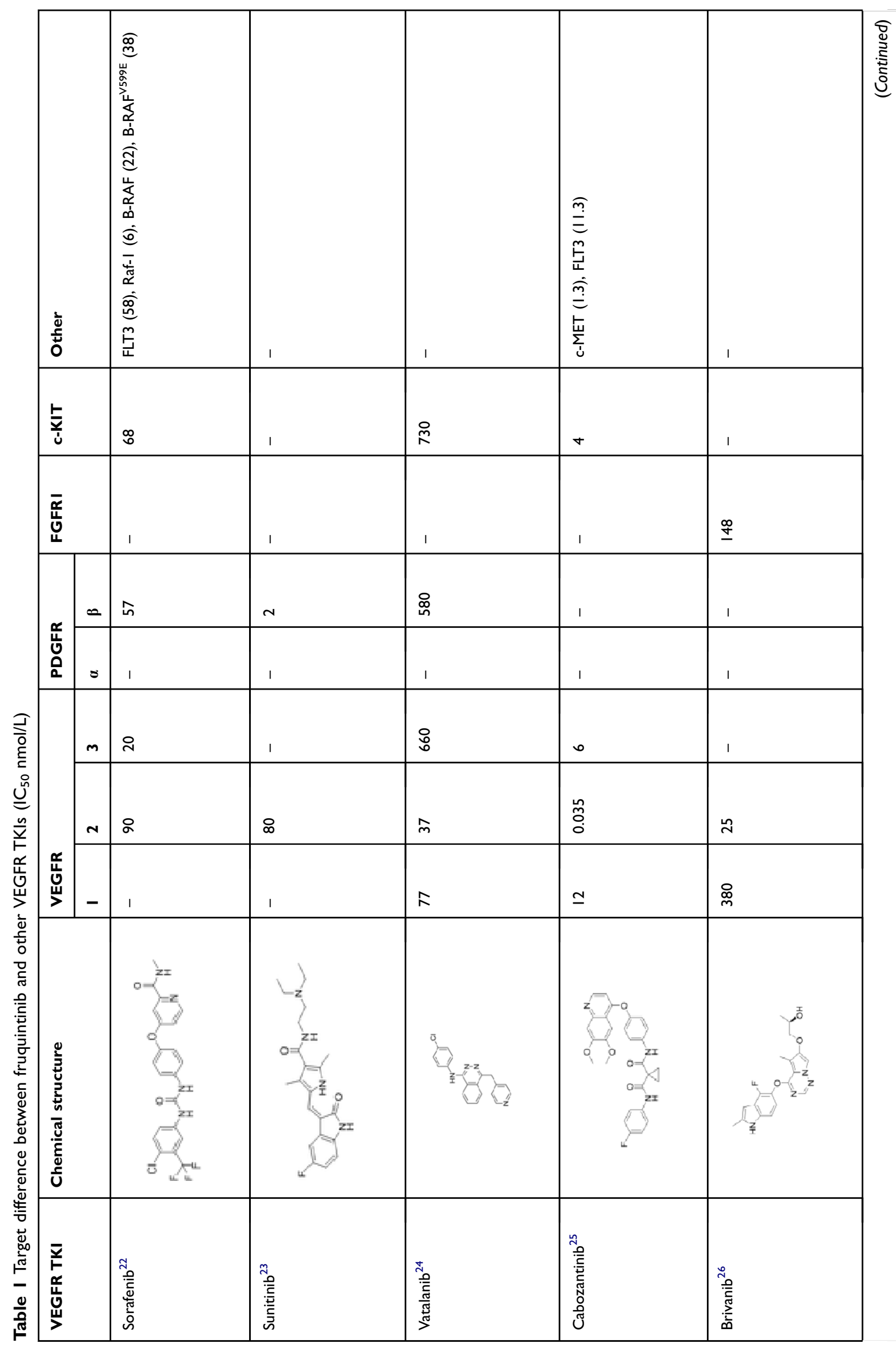




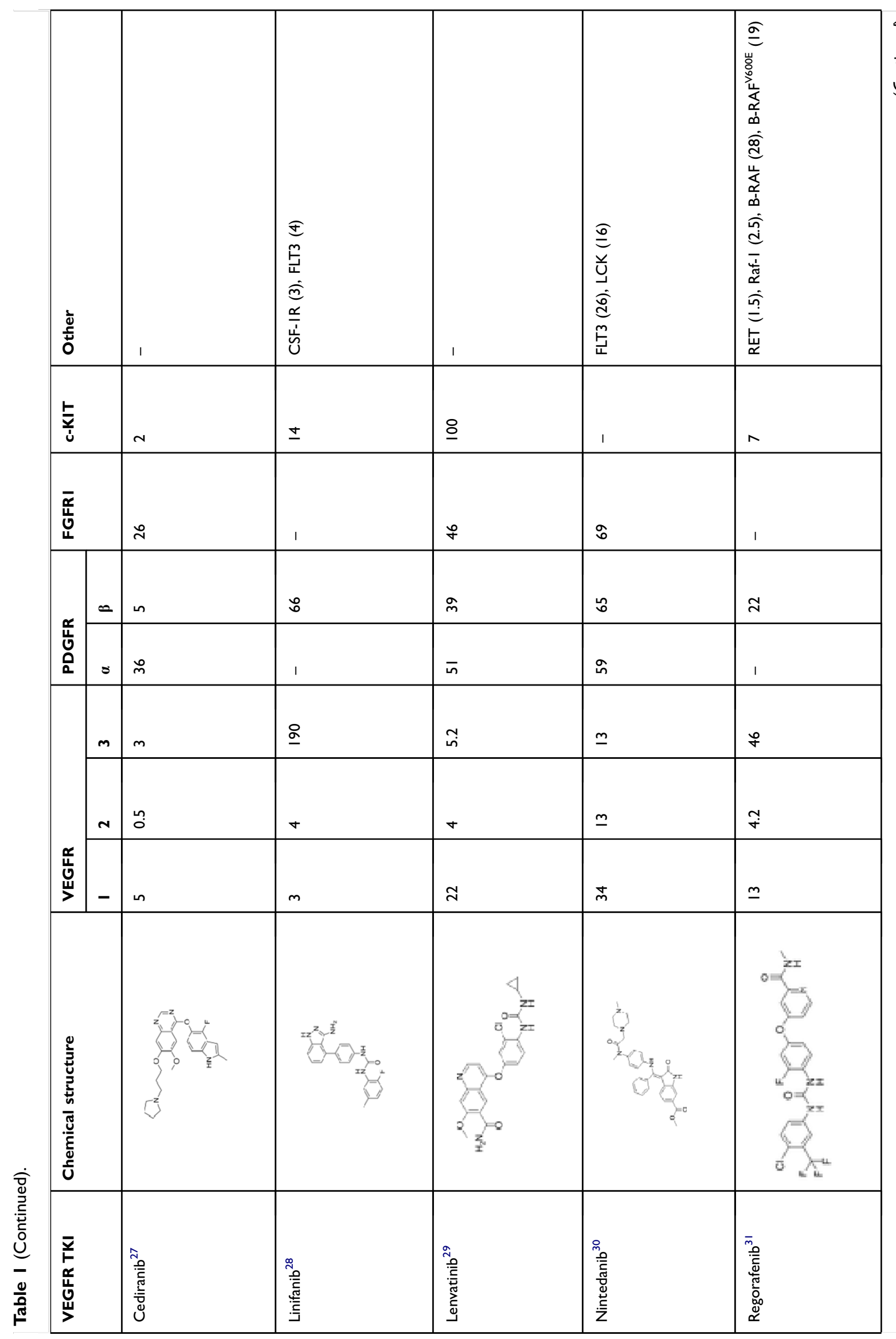




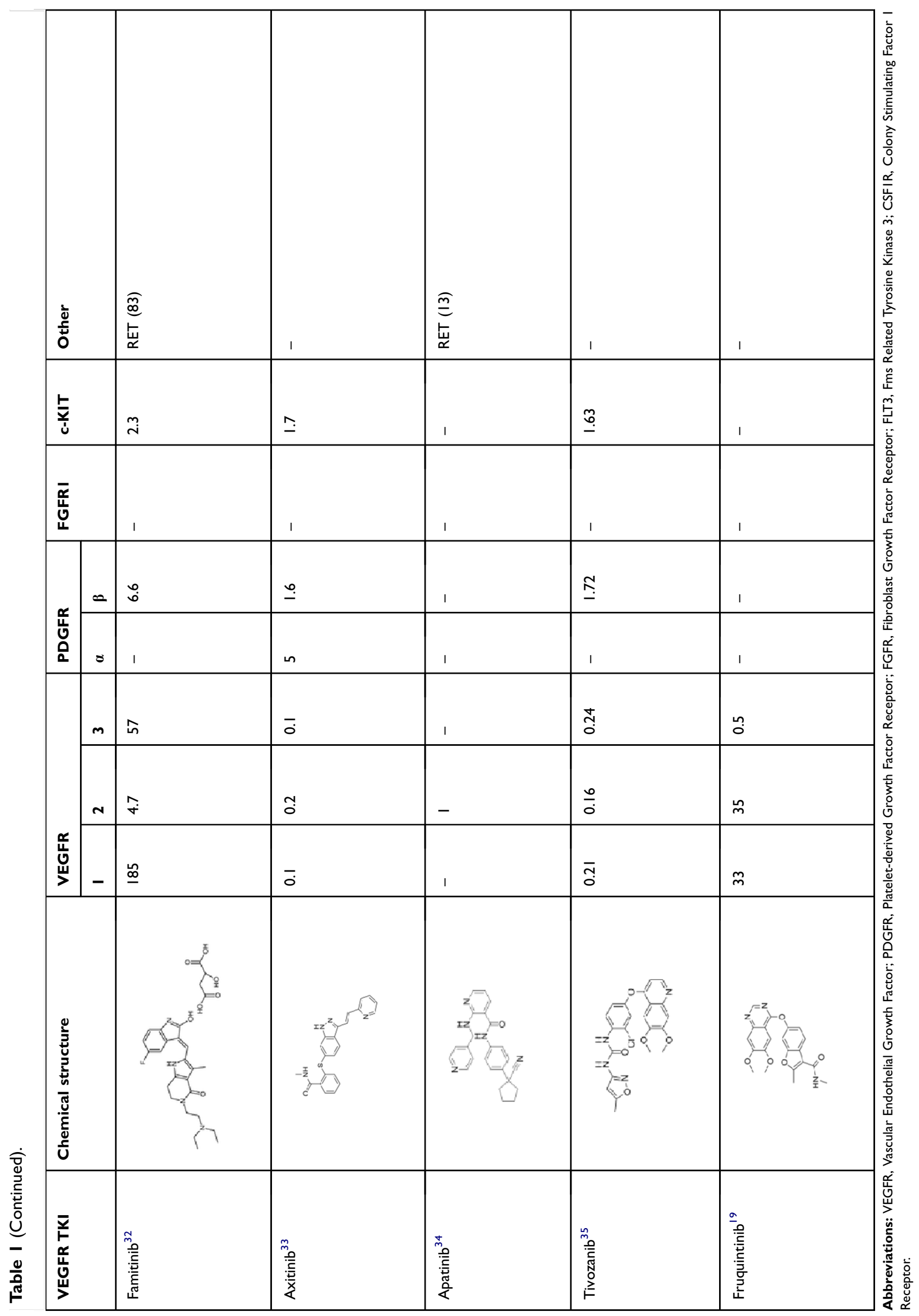


care (BSC) was given to all patients. As there are a limited number of targeted drugs approved for third- and post-line treatment of CRC, all three trials were conducted in patients who progressed after second-line standard therapy. Clinical trials to estimate the efficacy of fruquintinib in $\mathrm{mCRC}$ are summarized in Table 2.

In an open-label, single-arm phase $\mathrm{Ib}$ trial (NCT01975077), a total of 42 patients with mCRC were enrolled and received fruquintinib. The results showed that fruquintinib had a good efficacy profile. As the primary endpoint, the median PFS was 5.80 months (95\% CI 4.017.60). As the secondary endpoints, the median OS was 8.88 months (95\% CI 7.53-15.53), the objective response rate (ORR) was $9.5 \%$ and the disease control rate (DCR) was, $76.2 \%{ }^{39}$

In a subsequent randomized, double-blind, placebocontrolled phase II trial (NCT02196688), 71 patients were randomly assigned in a $2: 1$ ratio, to receive fruquintinib or placebo. As the primary endpoint, the median PFS was significantly improved with fruquintinib (4.73 months) versus placebo (0.09 months); (hazard ratio $(\mathrm{HR})=0.30$; $P<0.001)$. As the secondary endpoints, the DCR of the fruquintinib group increased significantly versus placebo $(68.1 \%$ vs $20.8 \%, \quad P<0.001)$, but the median OS (7.72 months vs 5.52 months; HR $=0.71 ; P=0.29$ ) and ORR $(2.1 \%$ vs $0 \%, P=0.45)$ did not increase. Failure to obtain significant differences in OS and ORR could be attributed to the small sample size in this trial. ${ }^{39}$

The FRESCO trial (NCT02314819) was a multicenter, randomized, double-blind and placebo-controlled phase III trial that further evaluated the efficacy and safety of fruquintinib. A total of 416 patients were randomized to receive fruquintinib or placebo in a 2:1 ratio. Patients who had previously been treated with VEGF or EGFR inhibitors were enrolled in the trial, but those who had received VEGFR inhibitors were excluded. Patients continued treatment until tumor progression, intolerable toxicity, or study withdrawal. The results demonstrated that fruquintinib was effective and safe in the treatment of mCRC. As the primary endpoint, the OS was significantly improved in the fruquintinib group compared with the placebo group (9.3 months vs 6.6 months; $\mathrm{HR}=0.65$; $P<0.001)$. As the secondary endpoints, the PFS (3.7 months vs 1.8 months; $P<0.001)$, ORR ( $4.7 \%$ vs $0.0 \% ; P=0.01)$ and DCR $(62.2 \%$ vs $12.3 \% ; P<0.001)$, were also significantly increased in the fruquintinib group compared with placebo. Notably, the OS with fruquintinib treatment was superior to placebo in almost all

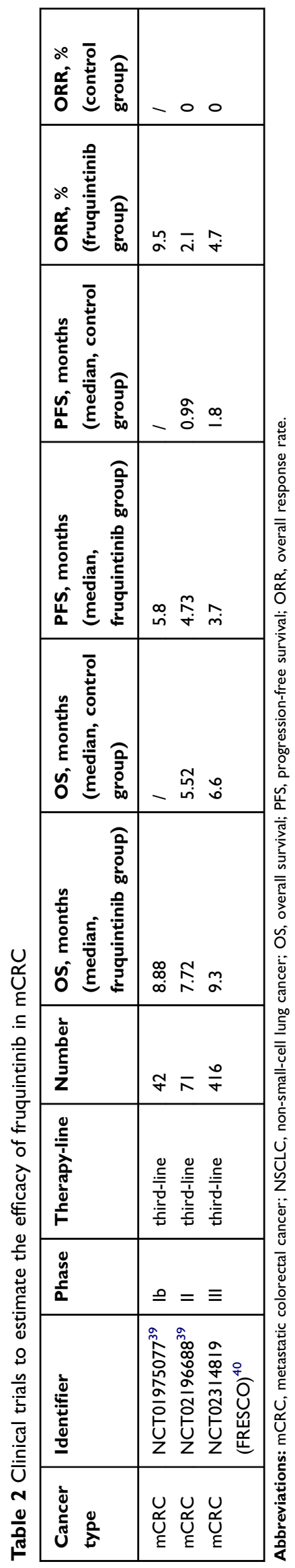


subgroups, especially in key subgroups including previous use of VEGF inhibitors [yes (HR 0.68, 95\% CI 0.45-1.03) vs no (HR $0.60,95 \%$ CI $0.45-0.80$ ); $P=0.72$ ], previous use of EGFR inhibitors [yes (HR $0.68,95 \%$ CI $0.35-1.30$ ) vs no (HR 0.62, 95\% CI 0.48-0.80); $P=0.75]$, and K-ras status [wild-type (HR $0.56,95 \%$ CI $0.40-0.78$ ) vs mutant (HR 0.75, 95\% CI 0.53-1.07); $P=0.26] .^{40}$

\section{Clinical trials of other anti-VEGFR TKIs: state of the art}

Over the past decade, attempts have been made to incorporate anti-VEGFR TKI into the treatment of mCRC. Unfortunately, most anti-VEGFR TKIs, which were successfully used to treat other malignancies, failed in mCRC. However, some anti-VEGFR TKIs such as regorafenib, famitinib, axitinib, apatinib, were effective in the treatment of mCRC. Clinical trials (Phase II/III) of anti-VEGFR TKIs that did not prove efficacy in mCRC are summarized in Table 3, and clinical trials (Phase II/III) that did prove efficacy in mCRC are summarized in Table 4.

\section{Sorafenib}

Sorafenib (NEXAVAR ${ }^{\circledR}$, Bayer, Leverkusen, Germany) is a first-generation small-molecule inhibitor of multiple targets, including VEGFR, platelet-derived growth factor (PDGFR), FLT3, KIT, Raf-1, B-RAF, and B-RAF. ${ }^{\text {V599E,22 }}$

Currently, one phase II trial investigating sorafenib plus cetuximab in patients with EGFR-expressing, KRAS-mutated mCRC has completed with results reported. A total of 30 patients who had progressed after 5-fluorouracil based regimens, were treated with sorafenib (400 mg orally twice daily) and cetuximab. An improvement in ORR was not observed (ORR $=0.0 \%$ ), therefore the trial did not meet its primary endpoint. This combination regimen was not recommended for previously treated mCRC. $^{41}$

\section{Sunitinib}

Sunitinib (SUTENT ${ }^{\circledR}$, Pfizer, NY, USA) is a first-generation, multi-targeted anti-VEGFR TKI with activity against VEGFR, PDGFR, KIT and Flt3. ${ }^{23}$

In a double-blind, randomized phase III trial, 768 treatment-naive patients with colorectal cancer were enrolled and randomized to receive FOLFIRI with sunitinib (37.5 mg orally once daily, in a 4-week-on/2-week-off regimen) or placebo. As the primary endpoint, PFS was shorter in the sunitinib group than in the placebo group, thus showing a lack of superiority for sunitinib with

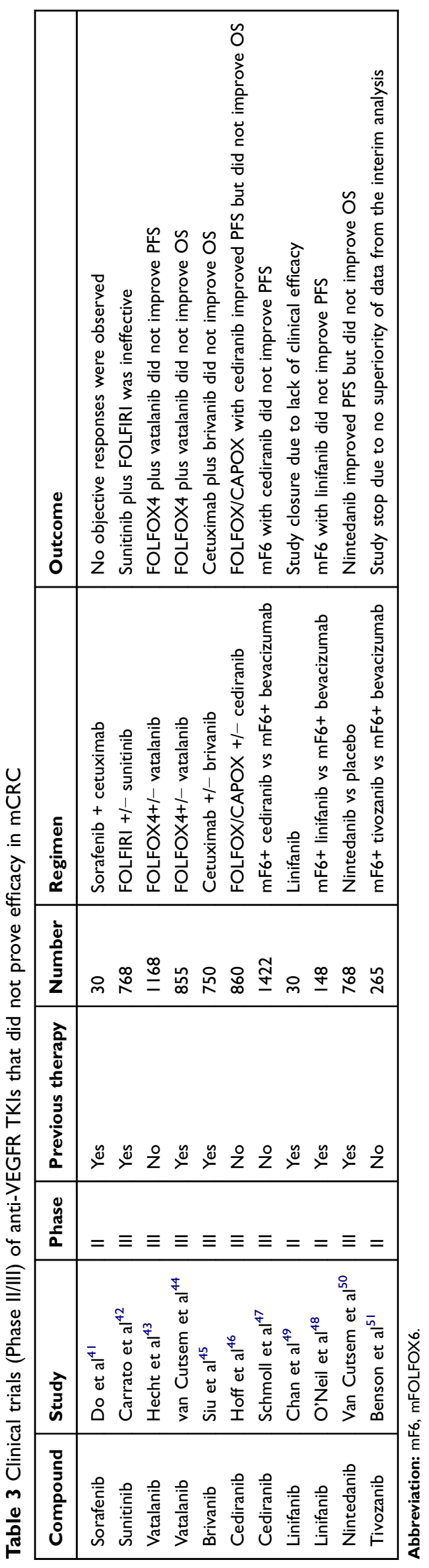




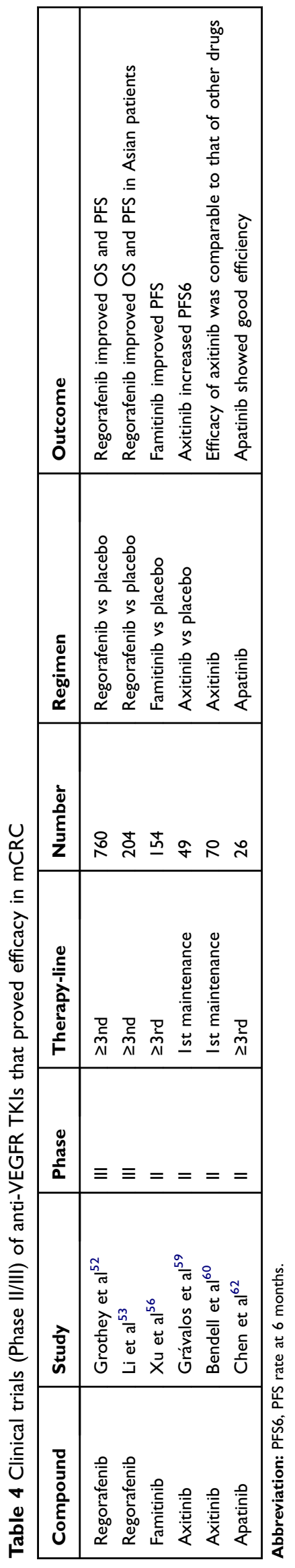

FOLFIRI (7.8 months vs 8.4 months; HR $=1.095$; $P=0.807$ ). More grade 3-4 adverse events and related deaths occurred in the sunitinib group than in the placebo group (12 vs 4). This trial, therefore, demonstrated that sunitinib in combination with FOLFIRI was not superior to FOLFIRI alone, and this combination regimen was not recommended for previously untreated mCRC. ${ }^{42}$

\section{Vatalanib}

Vatalanib (Novartis, Basel, Switzerland) is a first-generation anti-VEGFR TKI that inhibits tyrosine kinase signaling of all VEGFRs, PDGFR and KIT. ${ }^{24}$

Two randomized phase III trials (CONFIRM 1 and 2) with vatalanib were conducted in patients with mCRC. CONFIRM1 enrolled 1168 treatment-naive patients who were randomized to receive FOLFOX4 with vatalanib (1250 mg orally once daily) or placebo. The primary endpoint was PFS, and secondary endpoints included OS and ORR. The results showed that vatalanib did not significantly improve PFS (7.7 months vs 7.6 months; $P=0.118$ ), OS (21.4 months vs 20.5 months; $P=0.26)$, or ORR $(42 \%$ vs $46 \%) .{ }^{43}$ CONFIRM2 enrolled 855 pretreated patients. The primary endpoint was OS, and secondary endpoints included PFS and safety. Although median OS did not improve (13.1 months vs 11.9 months; $P=0.957$ ), median PFS was prolonged in the vatalanib group compared with placebo (5.6 months vs 4.2 months; $P=0.013$ ). Both CONFIRM1 and CONFIRM2 failed to reach their primary objectives. $^{44}$

\section{Brivanib}

Brivanib (BMS, NY, USA) is a first-generation oral antiVEGFR TKI with activity against VEGFRs and FGFRs. ${ }^{26}$

In a randomized phase III trial, 750 previously treated patients with wild-type K-RAS mCRC were randomized to receive cetuximab plus brivanib ( $800 \mathrm{mg}$ orally once daily) or placebo. Median OS did not improve with brivanib treatment compared with placebo (8.8 months vs 8.1 months; $P=0.12$ ), despite a significant increase in PFS (5.0 months vs 3.4 months; $P<0.001$ ). The trial did not meet the primary objective and this combination regimen was not recommended for patients with chemotherapy-refractory, wild-type K-RAS mCRC. ${ }^{45}$

\section{Cediranib}

Cediranib (AstraZeneca, London, UK) is a multi-targeted anti-VEGFR TKI that strongly blocks all VEGFRs, showing some modest activity against PDGFR, FGFR and $\mathrm{KIT}^{27}$ 
Phase II and III trials (HORIZON II and HORIZON III) were used to further evaluate the efficacy of cediranib. HORIZON II, a randomized phase III trial, enrolled 860 patients with previously untreated $\mathrm{mCRC}$ to receive cediranib $20 \mathrm{mg}$ (502 patients) or placebo (358 patients) combined with CAPOX/FOLFOX. As co-primary endpoints, PFS was prolonged in the cediranib group compared with placebo ( 8.6 months vs 8.3 months, $P=0.01$ ), but OS was not improved (19.7 months vs 18.9 months, $P=0.57){ }^{46}$ HORIZON III, a phase II/III randomized trial, enrolled 1422 patients with previously untreated $\mathrm{mCRC}$ to receive cediranib $20 \mathrm{mg}$ (709 patients) or bevacizumab (713 patients) combined with mFOLFOX6. As the primary endpoint, PFS did not improve in the cediranib group compared to the bevacizumab group $(9.9$ months vs 10.3 months, $P=0.11) .{ }^{47}$ As neither trial met their primary objective, a cediranib-containing regimen was not recommended in first-line treatment for patients with mCRC.

\section{Linifanib}

Linifanib (Abbott, IL, USA) is a multi-targeted antiVEGFR TKI that has inhibitory activity against VEGFRs, PDGFR $\beta$, KIT, FLT3 and CSF-1R. ${ }^{28}$

Two phase II studies were conducted to evaluate the efficacy and safety of linifanib in previously treated patients with mCRC. In one trial, a total of 148 patients were randomized in a 1:1:1 ratio, to receive mFOLFOX6 plus two doses of linifanib (7.5 and $12.5 \mathrm{mg}$, orally once daily) or bevacizumab. As the primary endpoint, PFS did not differ significantly between the three groups, even though a trend favoring bevacizumab over linifanib was observed. ${ }^{48}$ In the other phase II trial, a total of 30 patients received linifanib. The primary endpoint was ORR, with a goal of $10 \%$. Unfortunately, ORR $(0 \%)$ did not reach the primary aim, ${ }^{49}$ therefore, the efficacy of linifanib in mCRC needs to be further evaluated in more randomized phase III studies.

\section{Nintedanib}

Nintedanib (VARGATEF ${ }^{\circledR}$, BIBF, Mainz, Germany), is a first-generation multi-targeted anti-VEGFR TKI that potently blocks the proangiogenic pathways mediated by VEGFRs, PDGFRs, and FGFRs. ${ }^{30}$

The role of nintedanib in patients with refractory mCRC was investigated in the LUME-Colon 1 trial, a global and randomized phase III trial comparing nintedanib plus BSC versus placebo plus BSC. The trial did not meet its co-primary endpoints. The results showed that nintedanib did not improve OS but modestly increased PFS in the nintedanib group. ${ }^{50}$

\section{Tivozanib}

Tivozanib (FOTIVDA ${ }^{\circledR}$, Aveo, MA, USA) is a secondgeneration oral anti-VEGFR TKI with high selective inhibition of VEGFR-1, $-2,-3 .{ }^{35}$

In a phase II trial (BATON-CRC), previously untreated mCRC patients were randomized to receive FOLFOX6 plus tivozanib or bevacizumab. As the primary endpoint, PFS was similar in both groups $(9.4$ months vs 10.7 months, $P=0.706$ ). Data from the prespecified interim analysis did not show superiority and this led to discontinuation of the trial. ${ }^{51}$

\section{Regorafenib}

Regorafenib (STIVARGA ${ }^{\circledR}$, Bayer, Leverkusen, Germany) is a first-generation anti-VEGFR TKI that inhibits the activity of angiogenic (VEGFR1, $-2,-3$, TIE2), stromal (PDGFR, FGFR) and oncogenic (KIT, RET, RAF-1, BRAF, BRAF ${ }^{\mathrm{V} 600 \mathrm{E}}$ ) receptor tyrosine kinases. $^{31}$

The CORRECT trial led to the approval of regorafenib by the US Food and Drug Administration (FDA) in September 2012 and the European Medicines Agency in August 2013, for the treatment of mCRC. In this phase III trial, a total of 760 patients with refractory $\mathrm{mCRC}$ were randomized, in a 2:1 ratio, to receive regorafenib (160 $\mathrm{mg}$ orally once daily, in a 3week-on/1-week-off regimen) or placebo. Results showed significant improvements in the median OS (6.4 months vs 5.0 months; $\mathrm{HR}=0.77 ; P<0.01)$ and median PFS (1.9 months vs 1.7 months; HR $=0.49 ; P<0.0001)$. Patients receiving regorafenib had more grade 3-4 adverse events including hand-foot syndrome, fatigue, hypertension, diarrhea, and hepatotoxicity. ${ }^{52}$ The efficacy and safety of regorafenib were further evaluated in the phase III CONCUR trial. A total of 204 Asian patients with mCRC who progressed after standard second-line therapy were recruited. Patients were randomized to the regorafenib group and the placebo group, in a 2:1 ratio. Consistent with the results of the CORRECT trial, median OS (8.8 months vs 6.3 months; $\mathrm{HR}=0.55$; $P<0.001)$ and median PFS (3.2 months vs 1.7 months; HR $=0.31 ; P<0.0001)$ were significantly improved in the regorafenib group compared with the control group. The most common grade 3-4 adverse events were hand-foot skin reaction, hypertension and hepatotoxicity. ${ }^{53}$

\section{Famitinib}

Analog to sunitinib, famitinib (HengRui Medicine, Jiangsu, China) is a novel multi-targeted anti-VEGFR TKI. Famitinib potently inhibits VEGFR-2 and -3, PDGFR, c-KIT and RET, and has antitumor effects in a variety of solid tumors. ${ }^{54,55}$ 
In a multicenter, randomized, double-blind phase II trial, a total of 154 patients with refractory mCRC were enrolled. Patients were randomized to receive famitinib (25 mg orally once daily) or placebo, in a 2:1 ratio. As the primary endpoint, PFS in the famitinib group was longer than in the placebo group (2.8 months vs 1.5 months; HR $=0.58 ; P=0.0034)$. As secondary endpoints, DCR increased in the famitinib group compared with placebo (59.8\% vs $31.4 \% ; P=0.002)$, however, OS (7.4 months vs 7.2 months; $P=0.657)$ and ORR $(2.2 \%$ vs $0.0 \% ; P=0.540$ ) did not increase in the famitinib group. Famitinib showed a good safety profile. The most frequent grade 3-4 adverse events were hypertension, hand-foot syndrome, thrombocytopenia and neutropenia. ${ }^{56}$

Currently, only one phase II trial of famitinib has been conducted in mCRC and further clinical trials are necessary.

\section{Axitinib}

Axitinib (INLYTA ${ }^{\circledR}$, Pfizer, NY, USA) is a first-generation anti-VEGFR TKI with high potency against VEGFRs, PDGFR and c-KIT that has been approved by the FDA for the treatment of renal cell carcinoma. ${ }^{57,58}$

Two phase II trials were conducted to evaluate the efficacy and safety of axitinib as first-line maintenance therapy for mCRC. In one of the phase II trials, 49 patients who did not progress after $6-8$ months of first-line chemotherapy were enrolled and randomized to receive axitinib (5 mg orally twice a day) or placebo. As the primary endpoint, PFS at 6 months was increased in the axitinib group compared with placebo $(40.00 \%$ vs $8.33 \%$; $P=0.0141$ ). As secondary endpoints, median PFS showed an improvement in the axitinib group compared with placebo (4.9 months vs 3.1 months; $P=0.0116$ ), while median OS showed no statistically significant improvement (27.6 months vs 19.9 months; $P=0.3279$ ). The most common grade 3-4 adverse events were hypertension, diarrhea, and asthenia. ${ }^{59}$

In the other single-arm phase II trial, 70 patients who did not progress after 4 cycles of first-line mFOLFOX/ bevacizumab treatment were recruited and received maintenance therapy with axitinib. The initial oral dose of axitinib was $5 \mathrm{mg}$ twice daily, and after 2 weeks of administration, the dose was increased to $7 \mathrm{mg}$ twice daily in those patients that were able to tolerate axitinib well. As the primary endpoint, the median PFS was 8.3 months and the results showed that the efficacy of axitinib was comparable to that of other first-line maintenance drugs. ${ }^{60}$

Both trials have shown that axitinib is effective in the first-line maintenance therapy of $\mathrm{mCRC}$. Pending future large-scale, multicenter, Phase III clinical trials, axitinib is a promising candidate for maintenance therapy of mCRC.

\section{Apatinib}

Apatinib (AITAN ${ }^{\circledR}$, HengRui Medicine, Jiangsu, China) is a second-generation oral anti-VEGFR TKI with high selective inhibition of VEGFR-2. Apatinib also inhibits c-KIT, RET and c-SRC, and has a weak inhibitory effect on EGFR, HER-2 and FGFR. ${ }^{34}$ Apatinib was approved by CFDA for the treatment of advanced chemotherapy-refractory gastric cancer. ${ }^{61}$

In an open-label, single-arm phase II trial, a total of 26 patients who had undergone at least two standard treatments were enrolled to receive apatinib (500 mg orally once daily). The results showed that apatinib was effective in the treatment of refractory mCRC. The median PFS and OS were 3.9 months and 7.9 months, respectively. PFS was improved in the subgroup with $0-1$ performance status or no liver metastasis. All patients had dose reductions of apatinib due to adverse events. The most frequent grade 3-4 adverse events were hypertension, hand-foot syndrome, proteinuria, and diarrhea.

It is worth noting that the sample size in this trial is small and future studies in larger cohorts are necessary to prove the efficacy of apatinib. ${ }^{62}$

\section{Safety and tolerability of fruquintinib}

A 3-week-on/1-week-off regimen of $5 \mathrm{mg}$ fruquintinib once daily was recommended in the phase $\mathrm{Ib}$ trial and was used in the subsequent phase II-III trials.

In the phase Ib trial, treatment-emergent adverse events (TEAEs) associated with fruquintinib were observed in all 42 patients, of which $59.5 \%$ had grade 3-4 TEAEs. The most common (incidence $>5 \%$ ) grade 3-4 treatment-related TEAEs were hypertension (21.4\%), hand-foot skin reaction (HFSR, 9.5\%), diarrhea (9.5\%) and serum sodium decreased (7.1\%). Five patients (11.9\%) discontinued because of treatment-related TEAEs, and one patient (2\%) died of a potential treatment-related fatal hemoptysis. ${ }^{39}$

In the subsequent phase II trial, fruquintinib also showed good safety and manageable toxicity. In the fruquintinib group and the placebo group, the incidence of treatmentrelated TEAEs was $93.6 \%$ versus $58.3 \%$, respectively. The 
most common (incidence $>5 \%$ ) grade 3-4 treatment-related TEAEs were hypertension $(29.8 \%$ vs $0 \%)$ and HFSR $(14.9 \%$ vs $0 \%)$. The incidence of serious adverse events (SAEs) was similar in both groups (25.5\% vs $20.8 \%$ ). TEAEs leading to treatment modification (dose reduction or interruption) occurred in $61.7 \%$ of patients receiving fruquintinib, compared with $16.7 \%$ of patients in receiving placebo. The most common TEAEs leading to treatment modification were HFSR (17.0\%), hypertension $(12.8 \%)$ and diarrhea $(4.3 \%)$. The incidence of fatal TEAEs was similar in both groups (3 $[6 \%]$ patients in the fruquintinib group and $2[8 \%]$ patients in the placebo group). ${ }^{39}$

In the FRESCO trial, the safety and tolerability of fruquintinib were further evaluated. The incidence of treatment-related TEAEs was $95.7 \%$ in patients receiving fruquintinib versus $70.8 \%$ in patients receiving placebo. The most common treatment-related TEAEs were hypertension (55.4\% vs $15.3 \%$ ), HFSR (49.3 vs $2.9 \%$ ), proteinuria (42.1 vs $24.8 \%$ ), and dysphonia (36.0 vs $1.5 \%$ ). Most treatmentrelated TEAEs were grade 1-2 severity and could be controlled by treatment modification. Grade 3-4 treatment-related TEAEs occurred in $46 \%$ of patients in the fruquintinib group, compared with $7.3 \%$ in the placebo group. The most common (incidence $>5 \%$ ) grade 3-4 treatment-related TEAEs were hypertension $(21.2 \%$ vs $2.2 \%$ ) and HFSR (10.8\% vs $0 \%)$. Moreover, the incidence of SAEs was $15.5 \%$ in the fruquintinib group versus $5.8 \%$ in the placebo group. The incidence of treatment-related SAEs was $6.1 \%$ versus $1.5 \%$, respectively. TEAEs leading to treatment modification occurred in $47.1 \%$ of patients treated with fruquintinib, compared with $13.1 \%$ of patients in the placebo group. The most common TEAEs leading to treatment modification were HFSR (13.3\%), proteinuria (9.7\%), and decreased platelet counts (5.4\%). TEAEs leading to dose discontinuation occurred in $15.1 \%$ patients treated with fruquintinib, versus in $5.8 \%$ patients treated with placebo. In addition, the fatal TEAEs occurred in 9 (3.2\%) patients receiving fruquintinib and in $2(1.5 \%)$ patients receiving placebo. ${ }^{40}$ Treatment-related TEAEs in patients receiving fruquintinib in the FRESCO trial are shown in Figure 2.

Based on data from the above trials, fruquintinib showed an acceptable safety and tolerability profile in the treatment of $\mathrm{mCRC}$.

\section{Clinical trials in other solid tumors}

Fruquintinib is used not only to treat $\mathrm{mCRC}$, but also other solid tumors, such as lung cancer and gastric cancer. The status of most trials performed in other solid tumors is ongoing. Current clinical trials of fruquintinib in other solid cancers are summarized in Table 5.

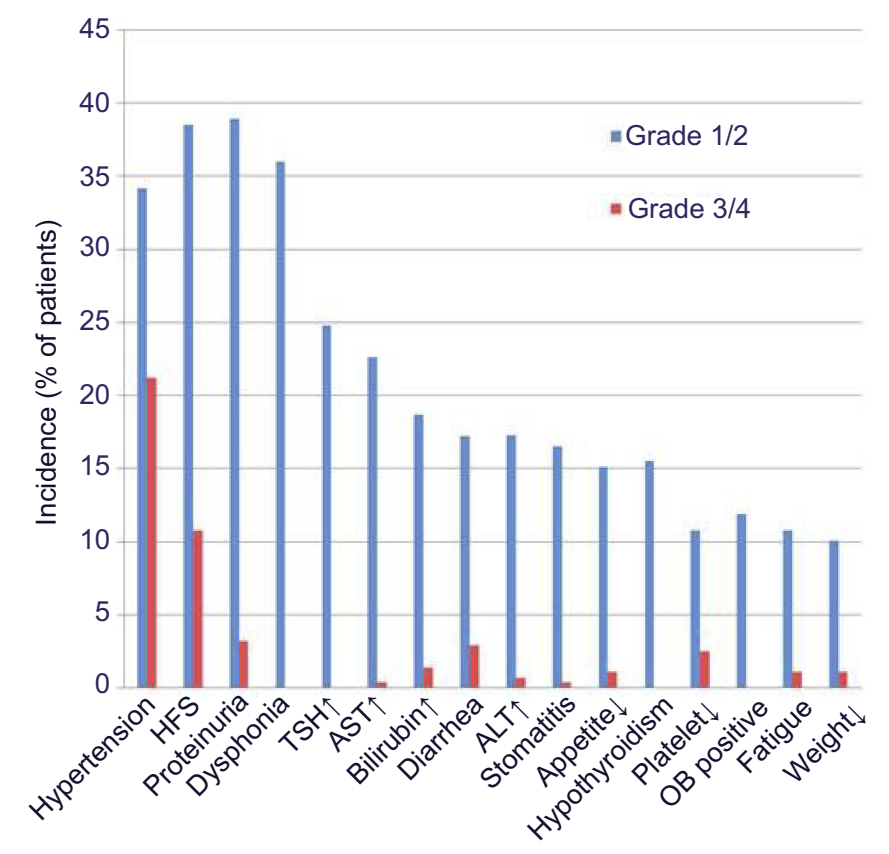

Figure 2 Treatment-related adverse events occurred in the fruquintinib group of the FRESCO trial. Notes: Adverse events reported in all patients $(n=416)$ in FRESCO trial.

Abbreviations: HFS, hand-foot skin reaction; TSH, thyroid stimulating hormone; AST, aspartate aminotransferase; ALT, alanine aminotransferase; OB, occult blood. 
Table 5 Current clinical trials of fruquintinib in other solid cancers

\begin{tabular}{|l|l|l|l|l|}
\hline Cancer type & Identifier & Phase & Enrollment & Regimen \\
\hline Advanced solid tumor & NCT0325I378 & I & Recruiting & Fruquintinib \\
Advanced solid tumor & NCT03903705 & I/II & Not recruiting & Fruquintinib plus sintilimab \\
Advanced NSCLC & NCT02976II6 & II & Not recruiting & Fruquintinib plus gefitinib \\
Advanced NSCLC & NCT02590965 & II & Completed & Fruquintinib vs placebo \\
Advanced NSCLC & NCT0269I299 & III & Completed & Fruquintinib vs placebo \\
Advanced gastric & NCT024I5023 & I/II & Completed & Fruquintinib plus paclitaxel \\
cancer & NCT03223376 & III & Recruiting & Fruquintinib plus paclitaxel vs placebo plus \\
Advanced gastric & (FRUTIGA) & & Paclitaxel \\
cancer & & & China \\
\hline
\end{tabular}

In advanced solid tumors, there are two ongoing trials. The first trial is a phase I trial (NCT03251378) to estimate PK, safety and tolerability of fruquintinib. This is the first clinical trial of fruquintinib in American patients following FDA acceptance of an investigational new drug application for fruquintinib. The second trial is a phase I/II trial (NCT03903705) to estimate the efficacy and safety of fruquintinib in combination with sintilimab.

In lung cancer, there are two completed trials and one ongoing trial. One of the completed trials is a phase II trial (NCT02590965) to estimate the efficacy and safety of fruquintinib for third-line treatment of advanced non-squamous non-small-cell lung carcinoma (NSCLC). 91 patients were randomly assigned to receive fruquintinib ( $5 \mathrm{mg}$ for 3 out of every 4 weeks) plus BSC or placebo plus BSC, in a 2:1 ratio. As the primary endpoint, median PFS was significantly improved in patients receiving fruquintinib compared with placebo (3.8 months vs 1.1 months; HR $=0.34 ; P<0.001)$. As secondary endpoints, 3 -and 6-month survival rates $(90.2 \%$ and $67.2 \%$ vs $73.3 \%$ and $58.8 \%)$, DRR $(13.1 \%$ vs $0 \% ; P=0.041)$ and DCR $(60.7 \%$ vs $13.3 \%$; $P<0.001)$ also showed statistically significant increases. Fruquintinib had an acceptable safety and tolerability profile, and the most common grade 3-4 TEAEs were hypertension $(8.2 \%)$, HFSR $(4.9 \%)$ and proteinuria $(4.9 \%){ }^{63}$ The second completed trial is the FALUCA phase III trial (NCT02691299). This trial was conducted to further evaluate the efficacy and safety of fruquintinib in 527 patients. Currently, the recruitment of patients is complete, and the results of this trial are expected to be announced soon. A phase II trial (NCT02976116) to evaluate the efficacy and safety of fruquintinib in combination with gefitinib, for the first-line treatment in patients with advanced non-squamous NSCLC harboring EGFR mutations, is ongoing.
In gastric cancer, there are two trials. One is a singlearm phase I/II trial (NCT02415023) to estimate PK and efficacy of fruquintinib in combination with paclitaxel. The other trial (FRUTIGA; NCT03223376) is a multicenter, randomized, double-blind phase III trial, to further evaluate the efficacy and safety of fruquintinib in combination with paclitaxel versus paclitaxel alone in secondline treatment of advanced gastric cancer.

\section{Discussion and future directions}

From data analysis of FRESCO trial, fruquintinib shows excellent efficacy and tolerable toxicity in patients with pretreated mCRC. Fruquintinib was approved by CFDA on September 4, 2018, for patients with mCRC who have progressed after at least two lines of prior treatment. Imminently, data from trials examining the efficacy of fruquintinib in the treatment of other solid tumors, such as advanced NSCLC and gastric cancer, will also be collected and analyzed.

Firstly, to gain a better understanding of the role of fruquintinib in the treatment of $\mathrm{mCRC}$, more clinical studies should be carried out. According to the clinical practice guidelines of National Comprehensive Cancer Network (NCCN), antiangiogenic drugs such as bevacizumab, aflibercept, ramucirumab and regorafenib can be used for mCRC treatment in different settings. In general, bevacizumab is used for first-line and second-line treatment, as well as maintenance therapy or cross-line therapy and aflibercept is used with FOLFIRI for second-line treatment. ${ }^{64,65}$ After first-line treatment with either bevacizumab, oxaliplatin or fluoropyrimidine, ramucirumab is also administered in combination with FOLFIRI or irinotecan-based regimen for second-line therapy. ${ }^{66}$ Regorafenib can be used for third- or later-line treatment in mCRC. ${ }^{67}$ However, drug resistance could occur at any 
time during $\mathrm{mCRC}$ treatment, therefore the development of new antiangiogenic TKIs is encouraged and welcomed. As a novel drug, fruquintinib improves PFS and OS in heavily pretreated $\mathrm{mCRC}$ patients and is approved as a third-line treatment for $\mathrm{mCRC}$. Additional studies are required to determine the efficacy of fruquintinib either in combination with chemotherapy for first- and secondline treatment or as a single-agent for first-line maintenance therapy.

A new strategy for the treatment of $\mathrm{mCRC}$ is the combination of antiangiogenic TKI and immunotherapy. The NCCN clinical guidelines have recommended anti-programmed death 1 (PD1) inhibitors for the treatment of $\mathrm{mCRC}$ with high microsatellite instability (MSI). ${ }^{68}$ PD1 inhibitors, nivolumab and pembrolizumab can improve PFS and ORR in patients with high-MSI mCRC. ${ }^{69,70}$ In order to obtain an optimized therapeutic effect of $\mathrm{mCRC}$ with high $\mathrm{MSI}$, a regimen of fruquintinib in combination with a PD-1 inhibitor might be adopted.

Secondly, fruquintinib may prove to be an effective treatment for other solid tumors in the future. In fact, many anti-angiogenic TKIs, such as regorafenib, apatinib and anlotinib, ${ }^{71}$ have been approved for the treatment of a variety of cancers. Further studies are required to demonstrate the efficacy of fruquintinib in the treatment of various other solid tumors. Furthermore, ongoing trials of fruquintinib in combination with chemotherapy or other TKI are being performed in a limited number of cancers, such as NSCLC and gastric cancer, and more studies should be conducted in other types of cancer.

Thirdly, differences in the efficacy and toxicity profiles of fruquintinib in different races remains unclear. Currently, all clinical trials on fruquintinib are conducted only in Chinese patients. More clinical trials should be conducted to estimate the efficacy and safety of fruquintinib in other races.

Finally, although fruquintinib has shown good efficacy in mCRC treatment, there are some issues to be explored and resolved before it can be widely used. One issue is the absence of molecular biomarkers that accurately predict the efficacy of fruquintinib. Some molecular biomarkers, such as ctDNA, ${ }^{62,72}$ activated circulating endothelial cells (aCECs) and neuropilin-1 (NRP-1), ${ }^{51,73}$ are tested to determine if the patient will benefit from TKI therapy. To date, there are still no proven molecular biomarkers to predict the efficacy of fruquintinib. In this case, trials aimed at identifying molecular biomarkers to predict the efficacy of fruquintinib should be designed and performed. Another key issue is the unknown mechanism of tumor resistance to fruquintinib in mCRC. More studies investigating potential mechanisms of resistance and most importantly, how to reverse resistance, should be conducted.

\section{Conclusion}

Antiangiogenesis plays an important role in the treatment of mCRC. At present, antiangiogenic drugs such as bevacizumab, aflibercept, ramucirumab and regorafenib are recommended by the NCCN guidelines for the treatment of mCRC. Fruquintinib is a potent and highly selective small-molecule inhibitor of VEGFR-1, -2 and -3 that plays an important role in third-line treatment of mCRC. In addition, fruquintinib is well tolerated and most TEAEs can be controlled or reversed by dose adjustment or drug intervention. In summary, fruquintinib is a novel anti-VEGFR TKI, and further research should aim to provide better guidance for its positioning in the mCRC strategic approach.

\section{Acknowledgment}

This work was supported by the National Youth Natural Science Foundation of China (Grant No. 81601692).

\section{Disclosure}

The authors report no conflicts of interest in this work.

\section{References}

1. Ferlay J, Soerjomataram I, Dikshit R, et al. Cancer incidence and mortality worldwide: sources, methods and major patterns in GLOBOCAN 2012. Int $J$ Cancer. 2015;136(5):E359-386. doi:10.1002/ijc. 29210

2. Arnold M, Sierra MS, Laversanne M, Soerjomataram I, Jemal A, Bray F. Global patterns and trends in colorectal cancer incidence and mortality. Gut. 2017;66(4):683-691. doi:10.1136/gutjnl-2015-310912

3. Van Cutsem E, Cervantes A, Nordlinger B, Arnold D; ESMO Guidelines Working Group. Metastatic colorectal cancer: ESMO clinical practice guidelines for diagnosis, treatment and follow-up. Ann Oncol. 2014;25(Suppl3):iii1-9. doi:10.1093/annonc/mdu203.

4. Tournigand $\mathrm{C}$, André T, Achille E, et al. FOLFIRI followed by FOLFOX6 or the reverse sequence in advanced colorectal cancer: a randomized GERCOR study. J Clin Oncol. 2004;22(2):229-237.

5. Colucci G, Gebbia V, Paoletti G, et al. Phase III randomized trial of FOLFIRI versus FOLFOX4 in the treatment of advanced colorectal cancer: a multicenter study of the Gruppo Oncologico Dell'Italia Meridionale [Southern Italy Oncology Group]. J Clin Oncol. 2005;23(22):4866-4875. doi:10.1200/JCO.2005.07.113

6. Yamazaki K, Nagase M, Tamagawa H, et al. Randomized phase III study of bevacizumab plus FOLFIRI and bevacizumab plus mFOLFOX6 as first-line treatment for patients with metastatic colorectal cancer (WJOG4407G). Ann Oncol. 2016;27(8):1539-1546. doi:10.1093/annonc/mdw141

7. Stintzing S, Modest DP, Rossius L, et al. FOLFIRI plus cetuximab versus FOLFIRI plus bevacizumab for metastatic colorectal cancer (FIRE-3): a post-hoc analysis of tumour dynamics in the final RAS wild-type subgroup of this randomised open-label phase 3 trial. Lancet Oncol. 2016;17(10):1426-1434. doi:10.1016/S1470-2045(16)30269-8 
8. Bellamy WT. Expression of vascular endothelial growth factor and its receptors in multiple myeloma and other hematopoietic malignancies. Semin Oncol. 2001;28(6):551-559. doi:10.1016/S0093-7754(01)90023-5

9. Tampellini M, Sonetto C, Scagliotti GV. Novel anti-angiogenic therapeutic strategies in colorectal cancer. Expert Opin Investig Drugs. 2016;25(5):507-520. doi:10.1517/13543784.2016.1161754

10. Tugues S, Koch S, Gualandi L, Li X, Claesson-Welsh L. Vascular endothelial growth factors and receptors: anti-angiogenic therapy in the treatment of cancer. Mol Aspects Med. 2011;32(2):88-111. doi:10.1016/j.mam.2011.04.004

11. Millauer B, Wizigmann-Voos S, Schnürch H, et al. High affinity VEGF binding and developmental expression suggest Flk-1 as a major regulator of vasculogenesis and angiogenesis. Cell. 1993;72 (6):835-846. doi:10.1016/0092-8674(93)90573-9

12. Tarallo V, De Falco S. The vascular endothelial growth factors and receptors family: up to now the only target for anti-angiogenesis therapy. Int J Biochem Cell Biol. 2015;64:185-189. doi:10.1016/j. biocel.2015.04.008

13. Zeng H, Dvorak HF, Mukhopadhyay D. Vascular permeability factor (VPF)/vascular endothelial growth factor (VEGF) peceptor-1 down-modulates VPF/VEGF receptor-2-mediated endothelial cell proliferation, but not migration, through phosphatidylinositol 3kinase-dependent pathways. J Biol Chem. 2001;276(29):2696926979. doi:10.1074/jbc.M103213200

14. De Falco S, Gigante B, Persico MG. Structure and function of placental growth factor. Trends Cardiovasc Med. 2002;12(6):241246. doi:10.1016/S1050-1738(02)00168-8

15. Bui HM, Enis D, Robciuc MR, et al. Proteolytic activation defines distinct lymphangiogenic mechanisms for VEGFC and VEGFD. $J$ Clin Invest. 2016;126(6):2167-2180.

16. Nasir A, Reising LO, Nedderman DM, et al. Heterogeneity of vascular endothelial growth factor receptors 1, 2, 3 in primary human colorectal carcinoma. Anticancer Res. 2016;36(6):2683-2696.

17. Kircher SM, Nimeiri HS, Benson AB 3rd. Targeting angiogenesis in colorectal cancer: tyrosine kinase inhibitors. Cancer J. 2016;22 (3):182-189. doi:10.1097/PPO.0000000000000192

18. Gotink KJ, Verheul HM. Anti-angiogenic tyrosine kinase inhibitors: what is their mechanism of action? Angiogenesis. 2010;13(1):1-14.

19. Sun Q, Zhou J, Zhang Z, et al. Discovery of fruquintinib, a potent and highly selective small molecule inhibitor of VEGFR 1, 2, 3 tyrosine kinases for cancer therapy. Cancer Biol Ther. 2014;15(12):16351645. doi:10.4161/15384047.2014.964087

20. Shirley M. Fruquintinib: first global approval. Drugs. 2018;78 (16):1757-1761. doi:10.1007/s40265-018-0998-z

21. Burki TK. Fruquintinib for previously treated metastatic colorectal cancer. Lancet Oncol. 2018;19(8):e388. doi:10.1016/S1470-2045(18) 30144-X

22. Wilhelm SM, Carter C, Tang L, et al. BAY 43-9006 exhibits broad spectrum oral antitumor activity and targets the RAF/MEK/ERK pathway and receptor tyrosine kinases involved in tumor progression and angiogenesis. Cancer Res. 2004;64(19):7099-7109.

23. Sun L, Liang C, Shirazian S, et al. Discovery of 5-[5-fluoro-2-oxo1,2-dihydroindol-(3Z)-ylidenemethyl]-2,4- dimethyl-1H-pyrrole-3carboxylic acid (2-diethylaminoethyl)amide, a novel tyrosine kinase inhibitor targeting vascular endothelial and platelet-derived growth factor receptor tyrosine kinase. J Med Chem. 2003;46(7):1116-1119.

24. Wood JM, Bold G, Buchdunger E, et al. PTK787/ZK 222584, a novel and potent inhibitor of vascular endothelial growth factor receptor tyrosine kinases, impairs vascular endothelial growth factor-induced responses and tumor growth after oral administration. Cancer Res. 2000;60(8):2178-2189.

25. You WK, Sennino B, Williamson CW, et al. VEGF and c-Met blockade amplify angiogenesis inhibition in pancreatic islet cancer. Cancer Res. 2011;71(14):4758-4768. doi:10.1158/0008-5472.CAN$10-2527$
26. Bhide RS, Cai ZW, Zhang YZ, et al. Discovery and preclinical studies of (R)-1-(4-(4-fluoro-2-methyl-1H-indol-5-yloxy)-5- methylpyrrolo[2,1-f][1,2,4]triazin-6-yloxy)propan- 2-ol (BMS-540215), an in vivo active potent VEGFR-2 inhibitor. J Med Chem. 2006;49 (7):2143-2146. doi:10.1021/jm0600592

27. Wedge SR, Kendrew J, Hennequin LF, et al. AZD2171: a highly potent, orally bioavailable, vascular endothelial growth factor receptor-2 tyrosine kinase inhibitor for the treatment of cancer. Cancer Res. 2005;65(10):4389-4400. doi:10.1158/0008-5472. CAN-04-4557

28. Albert DH, Tapang P, Magoc TJ, et al. Preclinical activity of ABT869, a multitargeted receptor tyrosine kinase inhibitor. Mol Cancer Ther. 2006;5(4):995-1006. doi:10.1158/1535-7163.MCT-05-0235

29. Matsui J, Yamamoto Y, Funahashi Y, et al. E7080, a novel inhibitor that targets multiple kinases, has potent antitumor activities against stem cell factor producing human small cell lung cancer H146, based on angiogenesis inhibition. Int $J$ Cancer. 2008;122(3):664-671. doi:10.1002/ijc. 23131

30. Hilberg F, Roth GJ, Krssak M, et al. BIBF 1120: triple angiokinase inhibitor with sustained receptor blockade and good antitumor efficacy. Cancer Res. 2008;68(12):4774-4782.

31. Wilhelm SM, Dumas J, Adnane L, et al. Regorafenib (BAY 734506): a new oral multikinase inhibitor of angiogenic, stromal and oncogenic receptor tyrosine kinases with potent preclinical antitumor activity. Int J Cancer. 2011;129(1):245-255.

32. Zhou A, Zhang W, Chang C, et al. Phase I study of the safety, pharmacokinetics and antitumor activity of famitinib. Cancer Chemother Pharmacol. 2013;72(5):1043-1053. doi:10.1007/s00280013-2282-y

33. Hu-Lowe DD, Zou HY, Grazzini ML, et al. Nonclinical antiangiogenesis and antitumor activities of axitinib (AG-013736), an oral, potent, and selective inhibitor of vascular endothelial growth factor receptor tyrosine kinases 1, 2, 3. Clin Cancer Res. 2008;14 (22):7272-7283. doi:10.1158/1078-0432.CCR-08-0652

34. Tian S, Quan H, Xie C, et al. YN968D1 is a novel and selective inhibitor of vascular endothelial growth factor receptor-2 tyrosine kinase with potent activity in vitro and in vivo. Cancer Sci. 2011;102(7):1374-1380.

35. Nakamura K, Taguchi E, Miura T, et al. KRN951, a highly potent inhibitor of vascular endothelial growth factor receptor tyrosine kinases, has antitumor activities and affects functional vascular properties. Cancer Res. 2006;66(18):9134-9142. doi:10.1158/0008-5472. CAN-05-4290

36. Gu Y, Wang J, Li K, et al. Preclinical pharmacokinetics and disposition of a novel selective VEGFR inhibitor fruquintinib (HMPL-013) and the prediction of its human pharmacokinetics. Cancer Chemother Pharmacol. 2014;74(1):95-115.

37. Cao J, Zhang J, Peng W, et al. A Phase I study of safety and pharmacokinetics of fruquintinib, a novel selective inhibitor of vascular endothelial growth factor receptor-1, -2 , and -3 tyrosine kinases in Chinese patients with advanced solid tumors. Cancer Chemother Pharmacol. 2016;78(2):259-269. doi:10.1007/s00280016-3089-4

38. Zhou S, Shao F, Xu Z, et al. A phase I study to investigate the metabolism, excretion, and pharmacokinetics of [14C]fruquintinib, a novel oral selective VEGFR inhibitor, in healthy Chinese male volunteers. Cancer Chemother Pharmacol. 2017;80(3):563-573. doi:10.1007/s00280-017-3394-6

39. $\mathrm{Xu} \mathrm{RH}$, Li J, Bai Y, et al. Safety and efficacy of fruquintinib in patients with previously treated metastatic colorectal cancer: a phase Ib study and a randomized double-blind phase II study. J Hematol Oncol. 2017;10(1):22.

40. Li J, Qin S, Xu RH, et al. Effect of fruquintinib vs placebo on overall survival in patients with previously treated metastatic colorectal cancer: the FRESCO randomized clinical trial. JAMA. 2018;319 (24):2486-2496. 
41. Do K, Cao L, Kang Z, et al. A phase II study of sorafenib combined with cetuximab in EGFR-expressing, KRAS-mutated metastatic colorectal cancer. Clin Colorectal Cancer. 2015;14(3):154-161.

42. Carrato A, Swieboda-Sadlej A, Staszewska-Skurczynska M, et al. Fluorouracil, leucovorin, and irinotecan plus either sunitinib or placebo in metastatic colorectal cancer: a randomized, phase III trial. $J$ Clin Oncol. 2013;31(10):1341-1347. doi:10.1200/JCO.2012.45.1930

43. Hecht JR, Trarbach T, Hainsworth JD, et al. Randomized, placebo-controlled, phase III study of first-line oxaliplatin-based chemotherapy plus PTK787/ZK 222584, an oral vascular endothelial growth factor receptor inhibitor, in patients with metastatic colorectal adenocarcinoma. J Clin Oncol. 2011;29(15):1997-2003. doi:10.1200/JCO.2010.29.4496

44. Van Cutsem E, Bajetta E, Valle J, et al. Randomized, placebo-controlled, phase III study of oxaliplatin, fluorouracil, and leucovorin with or without PTK787/ZK 222584 in patients with previously treated metastatic colorectal adenocarcinoma. J Clin Oncol. 2011;29(15):2004-2010.

45. Siu LL, Shapiro JD, Jonker DJ, et al. Phase III randomized, placebo-controlled study of cetuximab plus brivanib alaninate versus cetuximab plus placebo in patients with metastatic, chemotherapyrefractory, wild-type K-RAS colorectal carcinoma: the NCIC Clinical Trials Group and AGITG CO.20 Trial. J Clin Oncol. 2013;31(19):2477-2484. doi:10.1200/JCO.2013.49.0219

46. Hoff PM, Hochhaus A, Pestalozzi BC, et al. Cediranib plus FOLFOX/CAPOX versus placebo plus FOLFOX/CAPOX in patients with previously untreated metastatic colorectal cancer: a randomized, double-blind, phase III study (HORIZON II). J Clin Oncol. 2012;30 (29):3596-3603.

47. Schmoll HJ, Cunningham D, Sobrero A, et al. Cediranib with mFOLFOX6 versus bevacizumab with mFOLFOX6 as first-line treatment for patients with advanced colorectal cancer: a doubleblind, randomized phase III study (HORIZON III). J Clin Oncol. 2012;30(29):3588-3595. doi:10.1200/JCO.2012.42.5355

48. O'Neil BH, Cainap C, Van Cutsem E, et al. Randomized phase II open-label study of mFOLFOX6 in combination with linifanib or bevacizumab for metastatic colorectal cancer. Clin Colorectal Cancer. 2014;13(3):156-163. doi:10.1016/j.clcc.2014.04.001

49. Chan E, Goff LW, Cardin DB, et al. Phase II study of the multikinase inhibitor of angiogenesis, linifanib, in patients with metastatic and refractory colorectal cancer expressing mutated KRAS. Invest New Drugs. 2017;35(4):491-498. doi:10.1007/s10637-017-0458-8

50. Van Cutsem E, Yoshino T, Lenz HJ, et al. Nintedanib for the treatment of patients with refractory metastatic colorectal cancer (LUMEColon 1): a phase III, international, randomized, placebo-controlled study. Ann Oncol. 2018;29(9):1955-1963. doi:10.1093/annonc/ $\operatorname{mdx} 807$

51. Benson AB 3rd, Kiss I, Bridgewater J, et al. BATON-CRC: a phase II randomized trial comparing tivozanib plus mFOLFOX6 with bevacizumab plus mFOLFOX6 in stage IV metastatic colorectal cancer. Clin Cancer Res. 2016;22(20):5058-5067. doi:10.1158/1078-0432. CCR-16-0190

52. Grothey A, Van Cutsem E, Sobrero A, et al. Regorafenib monotherapy for previously treated metastatic colorectal cancer (CORRECT): an international, multicentre, randomised, placebo-controlled, phase 3 trial. Lancet. 2013;381(9863):303-312.

53. Li J, Qin S, Xu R, et al. Regorafenib plus best supportive care versus placebo plus best supportive care in Asian patients with previously treated metastatic colorectal cancer (CONCUR): a randomised, double-blind, placebo-controlled, phase 3 trial. Lancet Oncol. 2015;16 (6):619-629. doi:10.1016/S1470-2045(15)70156-7

54. Xie C, Zhou J, Guo Z, et al. Metabolism and bioactivation of famitinib, a novel inhibitor of receptor tyrosine kinase, in cancer patients. $\mathrm{Br} J$ Pharmacol. 2013;168(7):1687-1706. doi:10.1111/bph.12047

55. Chen Q, Tang L, Liu N, et al. Famitinib in combination with concurrent chemoradiotherapy in patients with locoregionally advanced nasopharyngeal carcinoma: a phase 1, open-label, dose-escalation study. Cancer Commun. 2018;38(1):66.
56. Xu RH, Shen L, Wang KM, et al. Famitinib versus placebo in the treatment of refractory metastatic colorectal cancer: a multicenter, randomized, double-blinded, placebo-controlled, phase II clinical trial. Chin J Cancer. 2017;36(1):97.

57. Rugo HS, Herbst RS, Liu G, et al. Phase I trial of the oral antiangiogenesis agent AG-013736 in patients with advanced solid tumors: pharmacokinetic and clinical results. J Clin Oncol. 2005;23 (24):5474-5483. doi:10.1200/JCO.2005.04.192

58. Bellesoeur A, Carton E, Alexandre J, Goldwasser F, Huillard O. Axitinib in the treatment of renal cell carcinoma: design, development, and place in therapy. Drug Des Devel Ther. 2017;11:28012811. doi:10.2147/DDDT.S109640

59. Grávalos C, Carrato A, Tobeña M, et al. A randomized phase II study of axitinib as maintenance therapy after first-line treatment for metastatic colorectal cancer. Clin Colorectal Cancer. 2018;17(2):e323e329.

60. Bendell JC, Joseph M, Barnes K, et al. A phase-2 trial of single agent axitinib as maintenance therapy following first-line treatment with modified FOLFOX/bevacizumab in patients with metastatic colorectal cancer. Cancer Invest. 2017;35(6):386-392. doi:10.1080/ 07357907.2017.1310221

61. Zhao D, Hou H, Zhang X. Progress in the treatment of solid tumors with apatinib: a systematic review. Onco Targets Ther. 2018;11:4137-4147. doi:10.2147/OTT.S172305

62. Chen X, Qiu T, Zhu Y, et al. A single-arm, phase II study of apatinib in refractory metastatic colorectal cancer. Oncologist. Epub 2019 Mar 15.

63. Lu S, Chang J, Liu X, et al. Randomized, double-blind, placebocontrolled, multicenter phase II study of fruquintinib after two prior chemotherapy regimens in Chinese patients with advanced nonsquamous Non-Small-Cell lung cancer. J Clin Oncol. 2018;36(12):12071217. doi: $10.1200 / \mathrm{JCO} .2017 .76 .7145$

64. Rosen LS, Jacobs IA, Burkes RL. Bevacizumab in colorectal cancer: current role in treatment and the potential of biosimilars. Target Oncol. 2017;12(5):599-610. doi:10.1007/s11523-017-0518-1

65. Fernández Montes A, Martínez Lago N, Covela Rúa M, et al. Efficacy and safety of FOLFIRI/aflibercept in second-line treatment of metastatic colorectal cancer in a real-world population: prognostic and predictive markers. Cancer Med. 2019;8(3):882889. doi:10.1002/cam4.1903

66. Noguerido A, Mulet-Margalef N, Matos I, et al. The safety of ramucirumab for the treatment of colorectal cancer. Expert Opin Drug Saf. 2018;17(9):945-951. doi:10.1080/14740338.2018.1506762

67. Bekaii-Saab T, Kim R, Kim TW, et al. Third- or later-line therapy for metastatic colorectal cancer: reviewing best practice. Clin Colorectal Cancer. 2019;18(1):e117-e129. doi:10.1016/j.clcc.2018.11.002

68. Cohen R, Pellat A, Boussion H, et al. Immunotherapy and metastatic colorectal cancers with microsatellite instability or mismatch repair deficiency. Bull Cancer. 2019;106(2):137-142. doi:10.1016/j. bulcan.2018.09.004

69. Mehrvarz Sarshekeh A, Overman MJ, Kopetz S. Nivolumab in the treatment of microsatellite instability high metastatic colorectal cancer. Future Oncol. 2018;14(18):1869-1874. doi:10.2217/fon-20170696

70. Marginean EC, Melosky B. Is there a role for programmed death ligand-1 testing and immunotherapy in colorectal cancer with microsatellite instability? Part II-the challenge of programmed death ligand-1 testing and its role in microsatellite instability-high colorectal cancer. Arch Pathol Lab Med. 2018;142(1):26-34. doi:10.5858/ arpa.2017-0041-RA

71. Shen G, Zheng F, Ren D, et al. Anlotinib: a novel multi-targeting tyrosine kinase inhibitor in clinical development. J Hematol Oncol. 2018;11(1):120. doi:10.1186/s13045-018-0664-7

72. Osumi H, Shinozaki E, Yamaguchi K, Zembutsu H. Clinical utility of circulating tumor DNA for colorectal cancer. Cancer Sci. 2019;110 (4):1148-1155. doi:10.1111/cas.2019.110.issue-4 
73. Liu Z, Wang J, Meng Z, et al. CD31-labeled circulating endothelial cells as predictor in anlotinib-treated non-small-cell lung cancer: analysis on ALTER-0303 study. Cancer Med. Epub 2018 Jun 1.

\section{Publish your work in this journal}

Cancer Management and Research is an international, peer-reviewed open access journal focusing on cancer research and the optimal use of preventative and integrated treatment interventions to achieve improved outcomes, enhanced survival and quality of life for the cancer patient.

Submit your manuscript here: https://www.dovepress.com/cancer-management-and-research-journal
The manuscript management system is completely online and includes a very quick and fair peer-review system, which is all easy to use. Visit http://www.dovepress.com/testimonials.php to read real quotes from published authors. 